\title{
Predictores de salud y pobreza en contagios de covid-19 en municipios rurales
}

\section{Predictors of health and poverty in covid-19 contages in rural municipalities}

\author{
DOI: $10.46932 / s f j d v 2 n 5-032$
}

Received in: Jun 1st, 2021

Accepted in: Sep 30th, 2021

\author{
Martha Jiménez Garcia \\ Instituto Politécnico Nacional \\ Maestría en Ciencias en Estudios Interdisciplinarios para Pequeñas y Medianas Empresas \\ E-mail: majimenez@ipn.mx \\ Keyla Dylorien Jiménez Santiago \\ Instituto Politécnico Nacional \\ Maestría en Ciencias en Estudios Interdisciplinarios para Pequeñas y Medianas Empresas \\ E-mail: kjimenezs1901@alumno.ipn.mx \\ Ingrid Anai Hernández Horta \\ Colegio de Postgraduados \\ Campos Montecillo \\ E-mail: hermandez.ingrid@colpos.mx
}

\section{RESUMEN}

El objetivo fue analizar datos de salud y pobreza para encontrar causas de contagio de COVID-19 en municipios rurales segmentados por hombres y mujeres. Se elaboraron dos modelos de regresión lineal múltiple por el método de mínimos cuadrados ordinarios, con datos de municipios rurales del país. En los resultados se encontró que en los hombres el contagio aumenta cuando tienen neumonía, asma, hipertensión, obesidad y presentan alguna carencia social, en cambio, en las mujeres el contagio aumenta con neumonía, diabetes, inmunosupresión, obesidad, presencia de carencia social y vivir en pobreza extrema. Se recomienda dar continuidad al análisis de la pobreza en municipios rurales incluyendo la movilidad de la población para evitar contagios. El trabajo tiene como limitación la omisión de la edad, y su originalidad es el análisis de las causas de contagio de COVID-19 en las comunidades rurales. Se concluye que es necesario dar prioridad en atención de salud a los hombres con COVID-19 con enfermedades de asma, obesidad e hipertensión, así como a las mujeres con inmunosupresión y que viven en pobreza extrema.

Palabras clave: Pobreza, Salud, COVID-19, México, Comunidad Rural

\section{ABSTRACT}

The objective was to analyze health and poverty data to find causes of contagion of COVID-19 in rural municipalities segmented by men and women. Two multiple linear regression models were developed using the ordinary least squares method, with data from rural municipalities in the country. In the results, it was found that in men the contagion increases when they have pneumonia, asthma, hypertension, obesity and present some social deprivation, on the other hand, in women the contagion increases with pneumonia, diabetes, immunosuppression, obesity, presence of social deprivation and live in extreme poverty. It is recommended to continue the analysis of poverty in rural municipalities including population mobility to avoid contagion. The work is limited by the omission of age, and its originality is the analysis 
of the causes of contagion of COVID-19 in rural communities. It is concluded that it is necessary to give priority in health care to men with COVID-19 with asthma, obesity and hypertension diseases, as well as to women with immunosuppression and living in extreme poverty.

Keywords: Poverty, Health, COVID-19, Mexico, Rural Community

\section{INTRODUCCIÓN}

La enfermedad por coronavirus, que se ha designado como COVID-19 por la Organización Mundial de la Salud (OMS), surgió en diciembre de 2019 en Wuhan, una ciudad de China. Esta se extendió a otras ciudades de China y, gradualmente, a otras partes del mundo (Ezeah et al., 2020). Asimismo, la OMS declaro el 30 de enero del 2020 la pandemia del COVID-19 y estableció la emergencia internacional como relevante a nivel mundial. Como consecuencia fueron creciendo globalmente los contagios por este virus.

Las comorbilidades tienen un alta probabilidad de contagio por COVID-19 (Pérez-Sastré et al., 2020; Moyce et al., 2020), afectando principalmente a las comunidades rurales con alto nivel de marginación (Meneses-Navarro et al., 2020; Díaz de León-Martínez et al., 2020; Vilar-Compte et al., 2020), así como mortalidad en personas mayores (Bodrud-Doza et al., 2020) y las comunidades que carecen de servicios de salud (Gutierrez, \& Bertozzi, 2020; Peters, 2020). De igual forma las estimaciones de causalidad muestran que la falta de acceso a servicios básicos conduce a un aumento de enfermedades transmisibles, incluido el COVID-19 (Anser et al., 2020).

Además hay que considerar que las personas en situación de pobreza no cumplen con las recomendaciones de quedarse en casa, pues tienen que salir a la calle a buscar sus ingresos económicos para sobrevivir (Bargain, \& Aminjonov, 2021). Sin embargo hace falta información más granular para analizar puntos críticos (Rivera-Hernandez et al., 2020), y poder apoyar a la población indígena en situación de pobreza, para crear conciencia de la enfermedad en la población que se encuentra en pobreza en comunidades rurales (Ezeah et al., 2020). Por otro lado, los segmentos más pobres de la población no pueden permitirse quedarse en casa y deben seguir trabajando, lo cual ha provocado una propagación más rápida del virus (Bargain, \& Aminjonov, 2021).

De acuerdo con la Secretaría de Salud del país, en México se tuvo el primer caso de COVID-19 el 27 de febrero de 2020 y posterior a ello, la mayoría de los casos de COVID-19 se ubicaron en la Ciudad de México. De igual forma, la edad promedio de los pacientes fue de 46 años y hubo una mayor incidencia en hombres $(58,18 \%)$ que en mujeres $(41,82 \%)$, además los pacientes fallecidos tenían una o múltiples comorbilidades, principalmente hipertensión $(45,53 \%)$, diabetes $(39,39 \%)$ y obesidad $(30,4 \%)$ (Suárez et al., 2020). En México al día 19 de noviembre del 2020 se presentaron 788,666 contagios y 109,939 
muertes causadas por la enfermedad del COVID-19, según datos publicados por la Dirección general de epidemiologia (Secretaría de Salud, 2020).

De igual forma, en México, el 50\% de la población indígena se encuentra en comunidades rurales (con menos de 2,500 habitantes), asimismo 11.8 millones de indígenas en México se encuentran en situación de pobreza y pobreza extrema, además 1.9 millones presentan rezago en acceso a servicios de salud, 9.4 millones en acceso a seguridad social (CONEVAL, 2019). Es aquí donde las cifras indican la necesidad de estudiar a las comunidades en situación de pobreza, siendo en este caso las causas de contagio por COVID-19.

Por ello, el objetivo de esta investigación es analizar datos de salud y pobreza para encontrar causas de contagio de COVID-19 en hombres y mujeres que viven en municipios rurales. Para dar respuesta a las interrogantes ¿Cuáles son las enfermedades que se relacionan con las causas de contagio del COVID19?, ¿Cuáles son los factores de pobreza que se relacionan con las causas de contagio del COVID-19?, enseguida se presenta la literatura científica relacionada con los contagios por COVID-19 en comunidades rurales.

\section{ANTECEDENTES DEL COVID-19}

En esta sección se presenta la literatura científica relacionaba con aspectos de comorbilidades asociadas al COVID-19 en comunidades rurales, el COVID-19 en comunidades rurales y la importancia del COVID-19 en la población.

\subsection{COMORBILIDADES ASOCIADAS AL COVID-19 EN COMUNIDADES RURALES}

En Estados Unidos un alto porcentaje de latinos ha resultado contagiado del COVID-19, debido a diversas carencias sociales y su alta comorbilidad, y varios han recurrido al uso de la medicina natural para prevenir enfermedades, dichos remedios naturales fueron aprendidos en sus países de origen para mantenerse saludables y calmarse, además, los hicieron sentirse protegidos contra las infecciones; estos remedios fueron el consumo de ajo, bicarbonato, vinagre, jugo de limón y líquidos calientes (Moyce et al., 2020).

Por otro lado en México, en la población de adultos mayores de 20 años, el asma y el tabaquismo no son factores de riesgo para contagiarse de COVID-19, sin embargo la población de adultos en México con desorden en el sistema inmunológico y con enfermedades cardiovasculares, metabólicas, inmunosupresión y EPOC, tienen una alta probabilidad de contagio por COVID-19 (Pérez-Sastré et al., 2020). 
Asimismo, en el país, el COVID-19 ha impactado desproporcionadamente a las comunidades rurales y de mayor edad, ya que más de un tercio de los adultos mayores han muerto de COVID-19 y se tienen tasas de mortalidad más altas en los adultos que viven en áreas rurales, pues la proporción de adultos mayores que dieron positivo en las áreas rurales fue del $21 \%$ en comparación con el 12,4\% en las áreas urbanas (Rivera-Hernandez et al., 2020).

De igual forma, las comorbilidades comunes como la hipertensión, la diabetes y las enfermedades cardiovasculares confieren el mayor riesgo de desarrollar COVID-19, lo cual podría resultar en una tasa más alta de enfermedad grave en caso de infección por COVID-19, lo que aumentaría la carga sobre los recursos ya limitados y generaría una mayor desigualdad (Arnold et al., 2020). En otro estudio para casos de contagios por COVID-19, se encontró que el 32\% de los pacientes tenían otros problemas de salud como diabetes, hipertensión y enfermedades cardiovasculares (Ezeah et al., 2020).

En un estudio realizado con adultos en Estados Unidos se encontró que los adultos con enfermedades crónicas eran más propensos a adherirse a las recomendaciones preventivas para reducir la propagación de COVID-19, de igual forma las personas adultas de mediana edad de Estados Unidos, no se unieron a las conductas preventivas de COVID-19, asimismo, estas personas presentaron una menor educación, menores ingresos, sin seguro, empleados, y residían en áreas rurales y suburbanas, además de no presentar ninguna enfermedad crónica física (Islam et al., 2021).

En Italia la infección por el COVID-19 comenzó a extenderse y afectó con mucha fuerza a la población, a pesar de la alta difusión, solo un subconjunto de pacientes desarrolló COVID-19 grave: alrededor del 25\% de ellos desarrolló una lesión renal aguda y un tercio de ellos murió. De igual forma se identificó que los pacientes de edad avanzada y los pacientes con comorbilidades altas tenían un mayor riesgo de COVID-19 grave; asimismo, en los pacientes hospitalizados por COVID-19, la hipertensión y la presión arterial baja en el momento de la presentación son factores de riesgo importantes para la mortalidad, de igual forma la reducción temprana de la terapia antihipertensiva puede mejorar los resultados en pacientes con infección por SARS-CoV-2 (Lanzani et al., 2021).

Además de aspectos de comorbilidad en las personas y derivado del COVID-19 y debido a la reducción de las actividades físicas y la ingesta activa de bocadillos, especialmente en los centros de cuarentena y aislamiento, se produce un aumento de peso que plantea complicaciones relacionadas con la diabetes (Mbunge, 2020). Lo que da muestra de la complejidad de la situación.

En esta sección se planteó la importancia de las comorbilidades en la población que reside en las comunidades rurales y su asociación al COVID-19, asimismo se presenta el uso de remedios caseros para el virus, así como porcentajes de las principales enfermedades como la diabetes, hipertensión y obesidad, entre otras. 


\subsection{EL COVID-19 EN COMUNIDADES RURALES}

El Covid-19 tuvo un impacto fuerte en América Latina y se extendió en las comunidades rurales, principalmente en los estados de Bolivia, Guatemala, México y Perú, siendo estos países vulnerables por presentar un alto nivel de marginación y diferencias culturales, por lo que se deben de tomar medidas para estas comunidades rurales de tal forma que se garantice la salud (Meneses-Navarro et al., 2020).

El gobierno federal de México, ha proporcionado acceso a la información de contagio y mortalidad de COVID-19, sin embargo, hace falta la información geográfica y de población más granular que permita una unidad de análisis más pequeña para identificar los puntos críticos (Rivera-Hernandez et al., 2020). Ya que la pandemia de COVID-19 podría tener efectos graves en el bienestar, la calidad de vida, los medios de subsistencia y la salud de todas las poblaciones, pero más significativamente en los más vulnerables(Díaz de León-Martínez et al., 2020).

El COVID-19 es un desafío para las comunidades indígenas de México, debido a las desigualdades sociales, por ejemplo en la zona rural de Oaxaca, el impacto de la pandemia en los pueblos indígenas tiene poco que ver con las tradiciones, creencias culturales o prácticas sociales locales, más bien, los desafíos planteados por Covid-19 son complejos y se basan en el apoyo estatal y federal limitado, la atención médica y la educación inadecuadas, así como los servicios deficientes de agua potable e Internet (Cohen, \& Mata-Sánchez, 2021).

Las personas más pobres presentan un menor cumplimiento de las recomendaciones de autoaislamiento, ya que tienen que continuar con las actividades generadoras de ingresos para sobrevivir durante la pandemia, pues las personas en áreas pobres tienden a ser trabajadores asalariados informales como jornaleros y comerciantes ambulantes (Bargain \& Aminjonov, 2021).

Son importantes los determinantes sociales de las comunidades en poblaciones de riesgo, según lecciones aprendidas del VIH y ébola; para disminuir los contagios de COVID-19 es necesario aumentar el acceso a los servicios de salud a la población vulnerable, así como darles un apoyo económico para hacer frente a la mitigación(Schwalbe et al., 2020).

En comunidades con desigualdad es imperativo proteger poblaciones vulnerables para evitar el contagio del COVID-19, en especial a grupos de familias con hijos que subsisten de la economía informal, ya que carecen las redes de seguridad social (Vilar-Compte et al., 2020). En esta sección se presentó el impacto que se encontró en la literatura científica del COVID-19 en las comunidades rurales, así como los desafíos que presentan dichas comunidades. 


\subsection{IMPORTANCIA DEL COVID-19 EN LA POBLACIÓN}

En Estados Unidos, la mayoría de la población latina, siguió las indicaciones impuestas por el gobierno, pese a que los cambios en su vida diaria les causaron preocupación y temor, y sintieron que parte de su comunidad no se estaba tomando el virus en serio; además, los latinos consultaban los informes de noticias nacionales y locales para obtener información y expresaron cautela ante los mensajes de las redes sociales, de igual forma muchos usaban remedios naturales para protegerse contra las infecciones(Moyce et al., 2020).

México puede necesitar estrategias de mitigación adicionales para limitar el riesgo de coinfección por COVID-19 e influenza entre los adultos mayores, es por ello que se necesitan esfuerzos de políticas para disminuir la propagación de COVID-19, particularmente entre las poblaciones vulnerables, y específicamente incluyendo a las comunidades rurales, asimismo el gobierno federal debe continuar fomentando el uso obligatorio de cubiertas faciales (Rivera-Hernandez et al., 2020). Además, los gobiernos locales de México deben trabajar con organizaciones comunitarias para identificar brechas en la atención médica para el tratamiento del Covid-19, entregar comidas y medicamentos, difundir información y promover el lavado e higiene de manos efectivos (Rivera-Hernandez et al., 2020).

El gobierno debe diseñar estrategias de comunicación en lenguas indígenas sobre la pandemia y las formas de cuidado, así como la elaboración de planes que aseguren el acceso a los servicios de diagnóstico y la atención hospitalaria, cuando sea necesario. De igual forma debe elaborar planes para proporcionar transporte terrestre y aéreo en casos graves, servicios hospitalarios gratuitos, acceso a medicamentos y equipo médico (incluida la terapia intensiva y los instrumentos de respiración asistida) en las comunidades rurales (Meneses-Navarro et al., 2020).

Los lugares rurales son más vulnerables en lo que respecta al estado de salud, ya que tienen menos consultorios médicos, más personas con una discapacidad de autocuidado y más residentes sin ningún seguro de salud público o privado. Además, la falta de seguro médico en comunidades rurales puede hacer que las personas eviten buscar tratamiento inmediato, empeorando su condición y aumentando el riesgo de propagación en la comunidad, lo mismo ocurre con el estrés financiero (Peters, 2020).

La mayor vulnerabilidad en las comunidades rurales es la falta de banda ancha para el acceso a Internet en general, esto limita la capacidad de los residentes de aprovechar los servicios de telemedicina para los enfermos, el teletrabajo para evitar despidos y la educación a distancia para los jóvenes (Peters, 2020). Asimismo, la conciencia y el conocimiento son requisitos esenciales para evitar el contagio del COVID-19, es por ello que las comunidades rurales corren un riesgo especial debido a su bajo nivel socioeconómico y su alto nivel de analfabetismo, por lo que es efectiva la comunicación interpersonal en 
la creación de conciencia y conocimiento sobre COVID-19 en las comunidades rurales (Ezeah et al., 2020)..

La edad también juega un papel en la vulnerabilidad de las personas al COVID-19. Las personas mayores son, más vulnerables al COVID-19, sin embargo, esto no significa que las personas más jóvenes no lo sean, ni es suficiente para concluir que las personas que no tienen otros problemas de salud no son vulnerables al COVID-19 (Ezeah et al., 2020). De igual forma en Brasil, para contener el virus, se realizó el aislamiento social obligatorio, lo cual provocó incertidumbres y miedo en personas mayores; por lo que es relevante realizar una planeación del área de salud para enfrentar el COVID-19 (De Castro et al., 2021).

Otros casos, como el de algunos países desarrollados y en específico, Nueva Zelanda y Australia, se observa que uno de cada cuatro neozelandeses y el 30\% de los australianos viven en zonas rurales y se presenta un mayor porcentaje de personas mayores que viven en estas áreas, además las necesidades de atención médica de estas comunidades son mayores que las de las poblaciones metropolitanas, sin embargo, el número de médicos, en particular especialistas, per cápita es menor (Arnold et al., 2020).

Es necesario evaluar de forma inmediata en las zonas rurales, la situación de COVID-19 y la capacidad de recuperación socioeconómica de una comunidad, para que los funcionarios de salud locales y estatales planifiquen y asignen sus recursos (Peters, 2020). Por ejemplo, en Sudáfrica, la pandemia de COVID-19 presenta efectos catastróficos en el sistema de salud, la sociedad y la economía, de igual forma sus continuos bloqueos nacionales han afectado el PIB del país y hacen imperativo que el gobierno reevalúe los efectos del bloqueo a medida que se desarrolla la pandemia (Mbunge, 2020).

De igual forma, en Sudáfrica el gobierno subestimó la gravedad de COVID-19, lo cual provocó un retraso en la acción contra la pandemia, sin embargo, cuando la OMS declaró medidas preventivas del COVID-19, sucedió que las autoridades sudafricanas implementaron distanciamiento físico, autoaislamiento, cierre de servicios no esenciales, escuelas, restricciones de viaje y cierres nacionales recurrentes para mitigar el impacto del COVID-19; esta reducción drástica de las actividades humanas e industriales durante el cierre provocaron problemas socioeconómicos, estallidos de incertidumbre, disturbios sociales, entre otros problemas sociales (Mbunge, 2020).

En la India se realizó un estudio y se encontró que el número acumulado de pacientes con COVID19 confirmados, depende de la población urbana, la población rural, el número de personas mayores de 50 años, la densidad de población y la tasa de pobreza, pues se encontró a través de un modelo por mínimos cuadrados ordinarios que el contagio de Covid-19 presentó un coeficiente de 0.25 para la tasa de pobreza, así como un coeficiente de 1.61 y de -0.33 para zonas urbanas y rurales respectivamente, y para la edad presentó un coeficiente de -0.03 (Pandey \& Saxena, 2020). 


\section{MÉTODO}

\subsection{PARTICIPANTES}

La muestra estuvo conformada por un total de 188 municipios rurales, dichos municipios tienen una población menor a 2500 habitantes, solo fueron considerados los municipios que presentaban personas con la enfermedad COVID-19 y que fueron diagnosticados de forma oficial por la Secretaría de Salud a través de un estudio de laboratorio del 1 de enero al 20 de noviembre de 2020.

\subsection{BASE DE DATOS}

Se analizaron los datos epidemiológicos relacionados al COVID-19, que se obtuvieron de la página web del gobierno de México (Secretaría de Salud, 2020). El período analizado fue entre el 1 de enero y el 20 de noviembre de 2020. De igual forma los datos epidemiológicos se unieron con indicadores de pobreza por entidad y municipio (CONEVAL, 2015).

\subsection{UNIÓN DE DATOS}

La base de datos de epidemiologia del COVID-19 fue descargada en un archivo de tipo "csv" y los datos de pobreza en un archivo Excel, ambos archivos fueron importados a una base de datos Sql Server versión 2019, en la cual se unieron ambas bases de datos, con el identificador entidad y municipio. En esta base de datos se seleccionaron los datos de las comunidades rurales y se agruparon por municipio y entidad y se realizó un conteo de variables de salud, y un promedio de porcentaje de la población sobre indicadores de pobreza. De igual forma se realizó una agrupación de datos por municipio, así como de hombres y mujeres con la enfermedad COVID-19.

\subsection{MODELOS PREDICTIVOS}

Se estimaron dos modelos predictivos mediante una regresión lineal múltiple por el método de mínimos cuadrados ordinarios. La variable dependiente fue personas contagiadas por municipio con COVID-19. Las variables independientes para el caso de los hombres en los municipios rurales con la enfermedad fueron neumonía, asma, inmunosupresión, hipertensión, obesidad, otro caso y carencias promedio de pobreza. Para el caso de las mujeres contagiadas las variables independientes fueron neumonía, diabetes, EPOC, inmunosupresión, cardiovascular, obesidad, renal crónica, tabaquismo, otro caso, razón ingreso de pobreza extrema, la carencia promedio de vulnerabilidad social, y la carencia promedio de ingreso de la línea de bienestar mínimo, las variables se presentan en la Figura 1 y se describen en la Tabla 1. Los modelos fueron validados mediante la evaluación de la hipótesis nula a través de la prueba $F$. También se calculó el coeficiente de determinación $R^{2}$. Para el análisis de datos y la 
elaboración de los modelos predictivos se utilizó Gretl v.2016 ${ }^{c}$. Los resultados de los coeficientes se consideraron significativos con $p \leq 0.05$.

Figura 1. Variables de estúdio

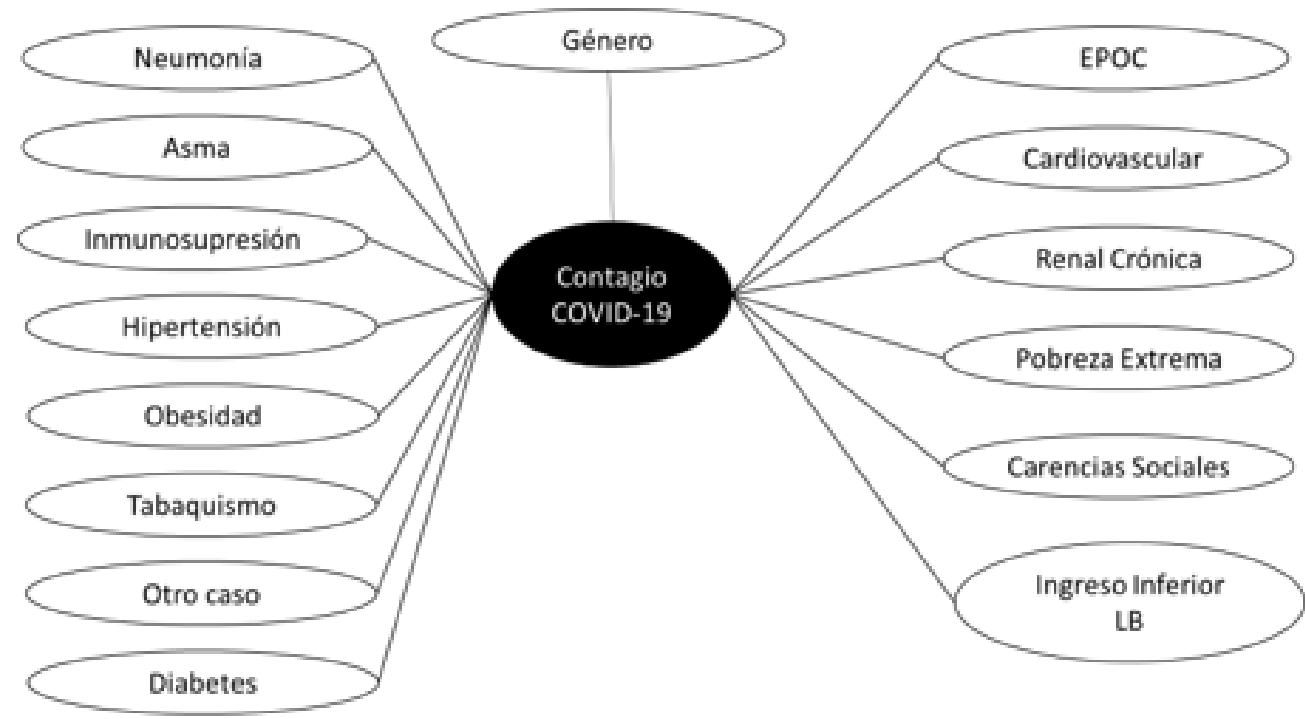

Fuente: Elaboración propia

Tabla 1. Descripción de variables incluidas en los modelos presentados, a partir de conteos por municipios rurales de México.

\begin{tabular}{|c|c|}
\hline Variable & Descripción \\
\hline $\begin{array}{l}\text { Contagio COVID-19 } \\
\text { (Y) }\end{array}$ & $\begin{array}{l}\text { Personas que tuvieron un resultado positivo a Sars-Cov-2 reportado por el } \\
\text { laboratorio de la Red Nacional de Laboratorios de Vigilancia Epidemiológica, } \\
\text { variable dependiente }\end{array}$ \\
\hline Género & Variable independiente de clasificación en los Modelos, Hombre y Mujer \\
\hline Neumonía $\left(\mathrm{X}_{1}\right)$ & $\begin{array}{l}\text { Paciente con COVID-19, que fue identificado con diagnosticó de neumonía, } \\
\text { variable independiente y dicotómica }(\mathrm{Si}=1 ; \mathrm{No}=0)\end{array}$ \\
\hline $\operatorname{Asma}\left(\mathrm{X}_{2}\right)$ & $\begin{array}{l}\text { Paciente con COVID-19, y con diagnóstico de asma, variable independiente y } \\
\text { dicotómica }(\mathrm{Si}=1 ; \mathrm{No}=0)\end{array}$ \\
\hline Inmunosupresión $\left(\mathrm{X}_{3}\right)$ & $\begin{array}{l}\text { Paciente con COVID-19, que fue identificado si el paciente presentaba } \\
\text { inmunosupresión, variable independiente y dicotómica }(\mathrm{Si}=1 ; \mathrm{No}=0)\end{array}$ \\
\hline Hipertensión $\left(\mathrm{X}_{4}\right)$ & $\begin{array}{l}\text { Paciente con COVID-19, y con diagnóstico de hipertensión, variable } \\
\text { independiente y dicotómica }(\mathrm{Si}=1 ; \mathrm{No}=0)\end{array}$ \\
\hline Obesidad $\left(\mathrm{X}_{5}\right)$ & $\begin{array}{l}\text { Paciente con COVID-19, y con diagnóstico de obesidad, variable independiente } \\
\text { y dicotómica }(\mathrm{Si}=1 ; \mathrm{No}=0)\end{array}$ \\
\hline Tabaquismo $\left(\mathrm{X}_{6}\right)$ & $\begin{array}{l}\text { Paciente con COVID-19, y se identifica con hábito de tabaquismo, variable } \\
\text { independiente y dicotómica }(\mathrm{Si}=1 ; \mathrm{No}=0)\end{array}$ \\
\hline Otro caso $\left(\mathrm{X}_{7}\right)$ & $\begin{array}{l}\text { Paciente con COVID-19, y que tuvo contacto con algún otro caso diagnosticado } \\
\text { con SARS CoV-2, variable independiente y dicotómica }(\mathrm{Si}=1 ; \mathrm{No}=0)\end{array}$ \\
\hline Diabetes $\left(\mathrm{X}_{8}\right)$ & $\begin{array}{l}\text { Paciente con COVID-19, y con diagnóstico de diabetes, variable independiente y } \\
\text { dicotómica }(\mathrm{Si}=1 ; \mathrm{No}=0)\end{array}$ \\
\hline EPOC $\left(X_{9}\right)$ & $\begin{array}{l}\text { Paciente con COVID-19, y con diagnóstico de EPOC, variable independiente y } \\
\text { dicotómica }(\mathrm{Si}=1 ; \mathrm{No}=0)\end{array}$ \\
\hline Cardiovascular $\left(\mathrm{X}_{10}\right)$ & $\begin{array}{l}\text { Paciente con COVID-19, y con diagnóstico de enfermedades cardiovasculares, } \\
\text { variable independiente y dicotómica }(\mathrm{Si}=1 ; \mathrm{No}=0)\end{array}$ \\
\hline Renal Crónica $\left(\mathrm{X}_{11}\right)$ & $\begin{array}{l}\text { Paciente con COVID-19, y con diagnóstico de insuficiencia renal crónica, } \\
\text { variable independiente y dicotómica }(\mathrm{Si}=1 ; \mathrm{No}=0)\end{array}$ \\
\hline Pobreza Extrema $\left(\mathrm{X}_{12}\right)$ & $\begin{array}{l}\text { Población que vive con menos de } 1.25 \text { dólares al día, variable independiente y } \\
\text { numérica expresada en porcentaje de la población. }\end{array}$ \\
\hline $\begin{array}{l}\text { Carencias Sociales } \\
\left(\mathrm{X}_{13}\right)\end{array}$ & $\begin{array}{l}\text { Población que presenta una o más carencias sociales (rezago educativo, acceso a } \\
\text { los servicios de salud, acceso a la seguridad social, calidad y espacios de la }\end{array}$ \\
\hline
\end{tabular}




\begin{tabular}{|l|l|}
\hline & $\begin{array}{l}\text { vivienda, servicios básicos en la vivienda y acceso a la alimentación), pero cuyo } \\
\text { ingreso es superior a la línea de bienestar, variable independiente y numérica } \\
\text { expresada en porcentaje de la población. }\end{array}$ \\
\hline $\begin{array}{l}\text { Ingreso Inferior LB } \\
\left(\mathrm{X}_{14}\right)\end{array}$ & $\begin{array}{l}\text { Población que no cuenta con los recursos suficientes para adquirir los bienes y } \\
\text { servicios que requiere para satisfacer sus necesidades (alimentarias y no } \\
\text { alimentarias), variable independiente y numérica expresada en porcentaje de la } \\
\text { población. }\end{array}$ \\
\hline
\end{tabular}

Fuente: Elaboración propia. Las variables de salud (Contagio COVID-19, Neumonía, Asma, Inmunosupresión, Hipertensión, Obesidad, Otro caso, Diabetes, EPOC, Cardiovascular y Renal Crónica) se obtuvieron de la Dirección General de Epidemiologia (Secretaría de Salud, 2020), y las variables de pobreza de la Metodología para la medición multidimensional de la pobreza en México (Consejo Nacional de Evaluación de la Política de Desarrollo Social (CONEVAL), 2014).

\subsection{MODELOS TEÓRICOS}

Para el caso de los hombres se tiene el modelo que se presenta en la ecuación 1, y para las mujeres se presenta la ecuación 2 .

$Y=\beta_{0}+\beta_{1} X_{1}+\beta_{2} X_{2}+\beta_{3} X_{3}+\beta_{4} X_{4}+\beta_{5} X_{5}+\beta_{7} X_{7}+\beta_{13} X_{13}$

$Y=\beta_{0}+\beta_{1} X_{1}+\beta_{3} X_{3}+\beta_{5} X_{5}+\beta_{6} X_{6}+\beta_{7} X_{7}+\beta_{8} X_{8}+\beta_{9} X_{9}+\beta_{10} X_{10}+\beta_{11} X_{11}+\beta_{12} X_{12}+\beta_{13} X_{13}+\beta_{14} X_{14}$

\section{RESULTADOS Y DISCUSIÓN}

En la Figura 2 se presentan los contagiados de COVID-19 por género en las comunidades rurales de México, y se aprecia claramente que la mayor cantidad de contagiados son los hombres (584), en tanto que las mujeres son un poco menos (517).

Figura 2. Contagiados de COVID-19 por género en comunidades rurales

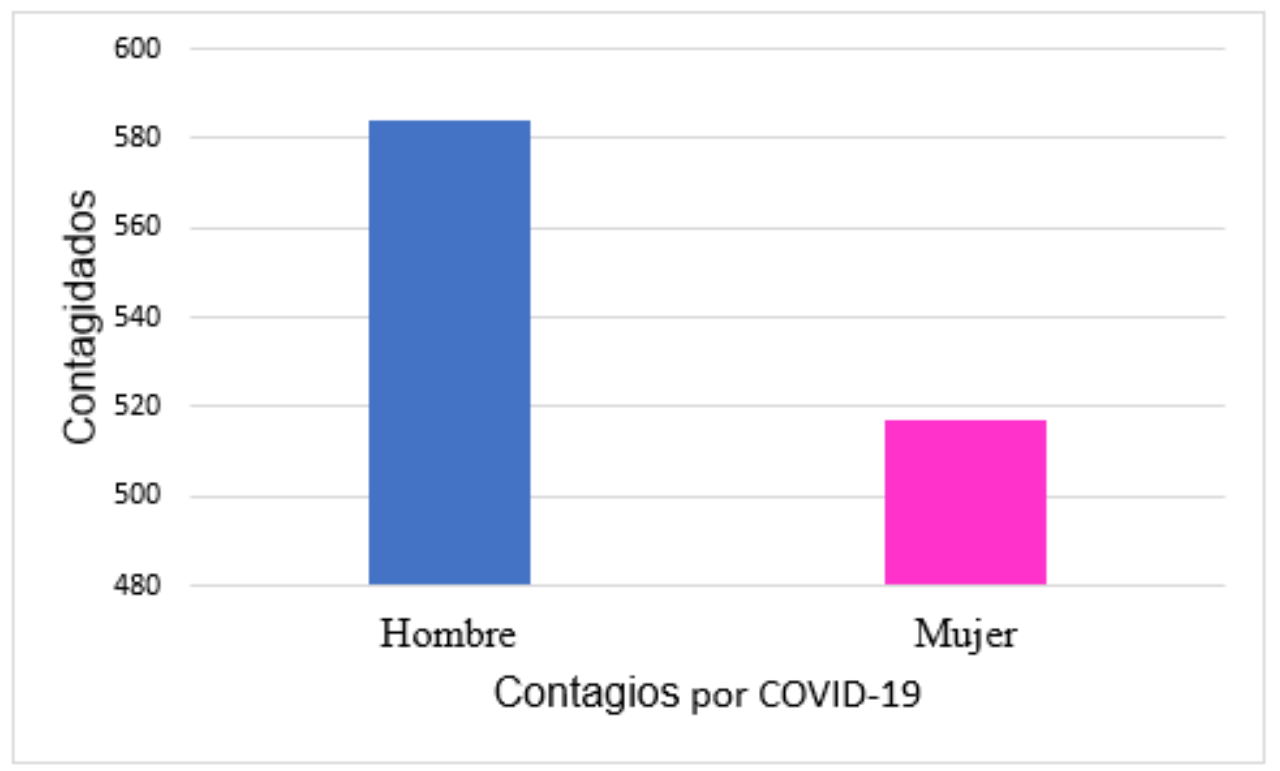

Fuente: Elaboración propia a partir de los resultados

En la Figura 3 se tiene; las comorbilidades de las personas que presentaron contagio por COVID19 por género, y se observa que ambos géneros tienen el mismo comportamiento, con una variante en la neumonía, tabaquismo y otro caso; asimismo para los hombres, la enfermedad que predomina es la 
neumonía e hipertensión, independientemente de otro caso (contacto con personas con COVID-19) y para las mujeres la enfermedad que predomina es la hipertensión y le sigue la diabetes, de igual forma independientemente de otro caso. Asimismo, en la Tabla 2 se presentan los estadísticos principales de las variables de estudio, que interactúan en el modelo y predicen el contagio por COVID-19 en hombres y mujeres en las comunidades rurales.

Figura 3. Comorbilidades de la población de los municipios rurales de contagio

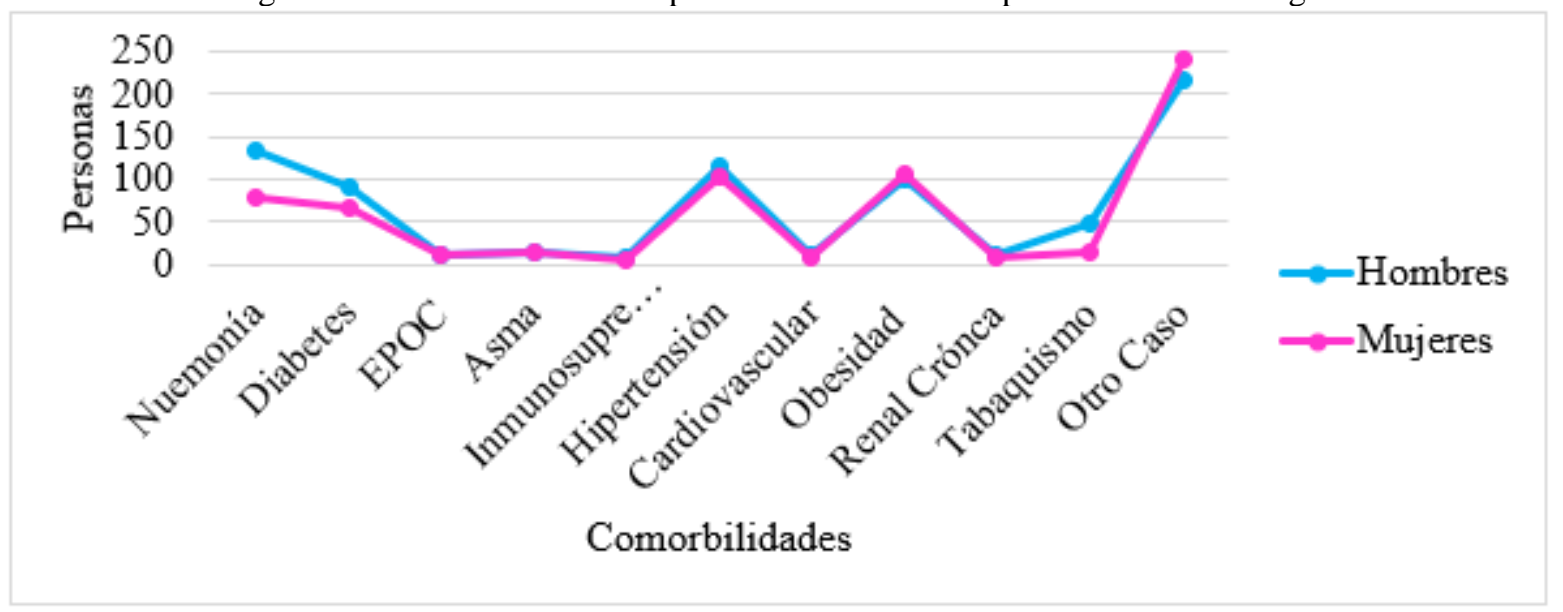

Fuente: Elaboración propia a partir de los resultados

Tabla 2. Estadísticos descriptivos para datos de los modelos de predicción de contagio de COVID-19 en hombres y mujeres en las comunidades rurales de México del 1 de enero y el 20 de noviembre de 2020.

\begin{tabular}{|l|l|l|l|l|l|}
\hline Variable & Media & Mediana & $\begin{array}{l}\text { Desviación } \\
\text { estándar }\end{array}$ & Mínimo & Máximo \\
\hline genero & 1.52 & 2.00 & 0.50 & 1.00 & 2.00 \\
\hline Neumonía & 0.54 & 0 & 1.32 & 0 & 15.0 \\
\hline Diabetes & 0.42 & 0 & 0.89 & 0 & 8.00 \\
\hline EPOC & 0.05 & 0 & 0.24 & 0 & 2.00 \\
\hline Asma & 0.09 & 0 & 0.34 & 0 & 3.00 \\
\hline Inmunosupresión & 0.03 & 0 & 0.17 & 0 & 1.00 \\
\hline Hipertensión & 0.56 & 0 & 1.18 & 0 & 15.0 \\
\hline Cardiovascular & 0.04 & 0 & 0.24 & 0 & 3.00 \\
\hline Obesidad & 0.54 & 0 & 1.45 & 0 & 20.0 \\
\hline Renal Crónica & 0.05 & 0 & 0.24 & 0 & 2.00 \\
\hline Tabaquismo & 0.15 & 0 & 0.65 & 0 & 9.00 \\
\hline Otro Caso & 1.27 & 0 & 3.88 & 0 & 39.0 \\
\hline Contagios & 2.98 & 1.00 & 6.84 & 1.00 & 87.0 \\
\hline Pobreza Extrema & 3.40 & 3.45 & 0.44 & 0 & 4.34 \\
\hline $\begin{array}{l}\text { Carencias Sociales de } \\
\text { Pobreza }\end{array}$ & 1.97 & 1.95 & 0.45 & 1.19 & 3.97 \\
\hline Ingreso menor LB & 2.36 & 2.46 & 0.62 & 0.78 & 4.30 \\
\hline
\end{tabular}

Fuente: Elaboración propia a partir de los resultados de los modelos 
En la Tabla 3 se presentan los modelos predictivos de contagio para hombres y mujeres respectivamente, ambos modelos son validados de forma estadística a través del valor $p$ de la prueba de $F$ y presentan aspectos importantes a considerar para tomar diferentes decisiones tanto de salud como de pobreza.

Tabla 3. Modelo predictivo de contagio de COVID-19 en hombres y mujeres Variable dependiente: contagio por COVID-19

\begin{tabular}{|c|c|c|c|c|}
\hline \multirow{2}{*}{ Variable } & \multicolumn{2}{|c|}{ Modelo Hombres } & \multicolumn{2}{|c|}{ Modelo Mujeres } \\
\hline & \multicolumn{4}{|c|}{ Coeficientes } \\
\hline constante & -2.27 & $* * *$ & -1.25 & $*$ \\
\hline Neumonía & 0.61 & $* * *$ & 0.93 & $* * *$ \\
\hline Asma & 3.45 & $* * *$ & & \\
\hline Inmunosupresión & -2.47 & $* * *$ & 9.21 & $* * *$ \\
\hline Hipertensión & 0.98 & $* * *$ & & \\
\hline Obesidad & 1.53 & $* * *$ & 1.45 & $* * *$ \\
\hline Tabaquismo & & & -1.26 & $* *$ \\
\hline Otro Caso & 0.79 & $* * *$ & 0.94 & $* * *$ \\
\hline Diabetes & & & 0.95 & $* * *$ \\
\hline EPOC & & & -1.93 & $* * *$ \\
\hline Cardiovascular & & & -1.42 & $*$ \\
\hline Renal Crónica & & & -1.25 & $* *$ \\
\hline Pobreza Extrema & & & 4.98 & $*$ \\
\hline Carencias sociales de pobreza & 1.23 & $* * *$ & 2.49 & $* * *$ \\
\hline Ingreso Inferior LB & & & -2.05 & $* * *$ \\
\hline \multicolumn{5}{|l|}{ Bondad de ajuste del modelo } \\
\hline & \multicolumn{2}{|c|}{$\begin{array}{l}\mathrm{R}^{2}=0.938, \mathrm{R}_{\mathrm{a}}^{2}=0.935 \\
\text { valor } p(\mathrm{de} F)=6.7 \mathrm{E}-106\end{array}$} & \multicolumn{2}{|c|}{$\begin{array}{l}\mathrm{R}^{2}=0.931, \mathrm{R}^{2}=0.925 \\
\text { valor } p(\mathrm{de} F)=5.14 \mathrm{e}-85\end{array}$} \\
\hline
\end{tabular}

*Significativo al 10\%, **Significativo al 5\%, *** Significativo al $1 \%$

Fuente: Elaboración propia a partir de los resultados de los modelos

Con base en los resultados que se presentan en la Tabla 3, para el caso del contagio por COVID19 en hombres, se tiene que la neumonía (0.61), asma (3.45), hipertensión (0.98), obesidad (1.53) y otro caso (0.79) son las variables que influyen en tener contagio, sin embargo, la inmunosupresión (-2.47) no influye para los hombres en adquirir contagio. De igual forma el asma y la obesidad son las variables que más influyen, por lo que habría que tener un mayor cuidado de estas enfermedades en los hombres. Asimismo, las carencias sociales de pobreza (1.23) también influyen en el contagio de COVID-19.

Asimismo, en los resultados que se presentan en la Tabla 3, para el caso del contagio por COVID19 en mujeres, se tiene que la neumonía (0.93), diabetes (0.95), inmunosupresión (9.21), obesidad (1.45) y otro caso (0.949 son las variables que influyen en tener contagio, sin embargo, EPOC (-1.93), cardiovascular $(-1.42)$, renal crónica $(-1.25)$ y tabaquismo $(-1,26)$ no influyen para las mujeres en adquirir contagio. De igual forma la inmunosupresión y la obesidad son las variables que más influyen, por lo que habría que tener un mayor cuidado de estas enfermedades en las mujeres. Asimismo, la pobreza extrema (4.98), así como las carencias sociales de pobreza (2.49) también influyen en el contagio de COVID-19, 
pero la variable ingreso inferior a la línea de bienestar (-2.05) no influye para las mujeres en adquirir el COVID-19.

El contagio de COVID-19 en las comunidades rurales se ha dado por diversas carencias sociales y por comorbilidades, lo cual coincide con Moyce et al. (2020), Arnold et al. (2020), entre otros. Asimismo, se concuerda con Pérez-Sastré et al. (2020) en que el tabaquismo no es un factor de riesgo para contagiarse de COVID-19, pero no se coincide con estos autores en cuanto al asma, pues en este caso esta enfermedad si es causa de contagio en las comunidades rurales para el caso de los hombres.

Las enfermedades transmisibles y la falta de saneamiento aumentan las cifras de pobreza con un valor de coeficiente de correlación de 0.587 p $<0.000$ y 0.487 p $<0.000$ respectivamente, mientras que el gasto en salud y el crecimiento económico disminuyen las tasas de pobreza con valores de correlación de $-0,551 \mathrm{p}<0,000 \mathrm{y}-0,567 \mathrm{p}<0,000$ respectivamente(Anser et al., 2020).

En un estudio similar de contagio de COVID-19 que fue elaborado por una regresión múltiple por el método de mínimos cuadrados ordinarios se presentó un coeficiente de 0.25 para la tasa de pobreza, sin embargo, el estudio no fue realizado por género, en tanto que en esta investigación se tiene un coeficiente de 4.98 para el contagio en mujeres en situación de pobreza extrema y de carencias sociales se tiene 1.23 y 2.49 para hombres y mujeres respectivamente. Se mencionó carencias sociales, debido a que esta variable representa los indicadores de pobreza, de tal forma que se aprecia que las mujeres con mayores carencias en las comunidades rurales son las de mayor riesgo de contagio por COVID-19 (Pandey, \& Saxena, 2020).

Los resultados encontrados refuerzan otra investigación que manifesta que los hombres tienen mayor riesgo que las mujeres en edades más avanzadas a contagiarse de COVID-19 (Cenobio García et al., 2021) y en esta investigación se incluyen los datos específicos por enfermedades, claro esta que falta profundizar tanto en temas por grupos de edad y género.

Para Suárez et al. (2020) las principales enfermedades en pacientes con COVID-19 son hipertensión, diabetes y obesidad, y para esta investigación son asma y obesidad para los hombres, así como inmunosupresión y obesidad para las mujeres. Por lo que se coincide con la enfermedad de obesidad.

\section{CONCLUSIONES}

Con base en los resultados presentados, las causas para que los hombres se contagien de COVID19 en las comunidades rurales son: 1) tener alguna enfermedad como neumonía, asma, hipertensión, obesidad, 2) haber estado en contacto con una persona con COVID-19, y 3) presentar alguna carencia social (rezago educativo, acceso a los servicios de salud, acceso a la seguridad social, calidad y espacios de la vivienda, servicios básicos en la vivienda y acceso a la alimentación). 
De igual forma con base en los resultados presentados, las causas para que las mujeres se contagien de COVID-19 en las comunidades rurales son: 1) tener alguna enfermedad como neumonía, diabetes, inmunosupresión y obesidad, 2) haber estado en contacto con una persona con COVID-19, 3) presentar alguna carencia social (rezago educativo, acceso a los servicios de salud, acceso a la seguridad social, calidad y espacios de la vivienda, servicios básicos en la vivienda y acceso a la alimentación) y 4) Pobreza extrema.

En las comunidades rurales el contagio de COVID-19 sucede por las enfermedades neumonía y obesidad, así como las carencias sociales de educación, servicios de salud, seguridad social, vivienda, y alimentación.

\section{AGRADECIMIENTOS}

Al Instituto Politécnico Nacional, proyecto SIP 20210776 "El Impacto del COVID-19 en las Comunidades Rurales con Pobreza". 


\section{REFERENCIAS}

Anser, M. K., Yousaf, Z., Khan, M. A., Nassani, A. A., Alotaibi, S. M., Qazi Abro, M. M., Vo, X. V., \& Zaman, K. (2020). Does communicable diseases (including COVID-19) may increase global poverty risk? A cloud on the horizon. Environmental Research, 187(May), 109668. https://doi.org/10.1016/j.envres.2020.109668

Arnold, R. H., Tideman, P. A., Devlin, G. P., Carroll, G. E., Elder, A., Lowe, H., Macdonald, P. S., Bannon, P. G., Juergens, C., McGuire, M., Mariani, J. A., Coffey, S., Faddy, S., Brown, A., Inglis, S., \& Wang, W. Y. S. (2020). Rural and Remote Cardiology During the COVID-19 Pandemic: Cardiac Society of Australia and New Zealand (CSANZ) Consensus Statement. Heart Lung and Circulation, 29(7), e88-e93. https://doi.org/10.1016/j.hlc.2020.05.001

Bargain, O., \& Aminjonov, U. (2021). Poverty and COVID-19 in Africa and Latin America. World Development, 142, 105422. https://doi.org/10.1016/j.worlddev.2021.105422

Bodrud-Doza, M., Shammi, M., Bahlman, L., Islam, A. R. M. T., \& Rahman, M. M. (2020). Psychosocial and Socio-Economic Crisis in Bangladesh Due to COVID-19 Pandemic: A Perception-Based Assessment. Frontiers in Public Health, 8(June). https://doi.org/10.3389/fpubh.2020.00341

Cenobio García, F. de J., Casillas Enríquez, J. D., Cázquez Sandrin, G., Garza Saldaña, O. F., \& Benítez Herrera, A. E. (2021). Características clínicas y epidemiológicas de la COVID-19 en las regiones indígenas de México Clinical and epidemiological characteristics of COVID-19 in the indigenous regions of Mexico. South Florida Journal of Development, 2(4), 5068-5080. https://doi.org/10.46932/sfjdv2n4-009

Cohen, J. H., \& Mata-Sánchez, N. D. (2021). Challenges, inequalities and COVID-19: Examples from indigenous Oaxaca, Mexico. Global Public Health. https://doi.org/10.1080/17441692.2020.1868548

CONEVAL. (2015). CONSULTA DINÁMICA DE RESULTADOS DE POBREZA A NIVEL MUNICIPIO 2010 Y 2015. https://www.coneval.org.mx/Medicion/Paginas/consulta_pobreza_municipal.aspx

Consejo Nacional de Evaluación de la Política de Desarrollo Social (CONEVAL). (2014). Metodología para la medición multidimensional de la pobreza en México. https://www.coneval.org.mx/InformesPublicaciones/InformesPublicaciones/Documents/Metodologiamedicion-multidimensional-3er-edicion.pdf

De Castro, L. P., De Souza, L. A., Da Fonseca, H. G., Modesto, J. G., Pernambuco, M. L., Lima, C. M. G., \& Pagnossa, J. P. (2021). Psychosocial impact of COVID-19 on the Brazilian elderly population. South Florida Journal of Development, 2(4), 5259-5267. https://doi.org/10.46932/sfjdv2n4-023

Díaz de León-Martínez, L., de la Sierra-de la Vega, L., Palacios-Ramírez, A., Rodriguez-Aguilar, M., \& Flores-Ramírez, R. (2020). Critical review of social, environmental and health risk factors in the Mexican indigenous population and their capacity to respond to the COVID-19. Science of the Total Environment, 733, 139357. https://doi.org/10.1016/j.scitotenv.2020.139357

Ezeah, G., Ogechi, E. O., Ohia, N. C., \& Celestine, G. V. (2020). Measuring the effect of interpersonal communication on awareness and knowledge of COVID-19 among rural communities in Eastern Nigeria. Health Education Research, 35(5), 481-489. https://doi.org/10.1093/her/cyaa033

Gutierrez, J. P., \& Bertozzi, S. M. (2020). Non-communicable diseases and inequalities increase risk of death among COVID-19 patients in Mexico. PLoS ONE, $15(10$ October), 1-11. https://doi.org/10.1371/journal.pone.0240394 
Islam, J. Y., Vidot, D. C., \& Camacho-Rivera, M. (2021). Determinants of COVID-19 preventive behaviours among adults with chronic diseases in the USA: an analysis of the nationally representative COVID-19 impact survey. BMJ Open, 11(2), e044600. https://doi.org/10.1136/bmjopen-2020-044600

Lanzani, C., Simonini, M., Arcidiacono, T., Messaggio, E., Bucci, R., Betti, P., Avino, M., Magni, G., Maggioni, C., Conte, C., Querini, P. R., Ciceri, F., Castagna, A., Vezzoli, G., \& Manunta, P. (2021). Role of blood pressure dysregulation on kidney and mortality outcomes in COVID - 19. Kidney, blood pressure and mortality in SARS - CoV - 2 infection. 0123456789. https://doi.org/10.1007/s40620-021-00997-0

Mbunge, E. (2020). Effects of COVID-19 in South African health system and society: An explanatory study. Diabetes and Metabolic Syndrome: Clinical Research and Reviews, 14(6), 1809-1814. https://doi.org/10.1016/j.dsx.2020.09.016

Meneses-Navarro, S., Freyermuth-Enciso, M. G., Pelcastre-Villafuerte, B. E., Campos-Navarro, R., MeléndezNavarro, D. M., \& Gómez-Flores-Ramos, L. (2020). The challenges facing indigenous communities in Latin America as they confront the COVID-19 pandemic. International Journal for Equity in Health, 19(1), 19-21. https://doi.org/10.1186/s12939-020-01178-4

Moyce, S., Velazquez, M., Claudio, D., Thompson, S., Metcalf, M., Aghbashian, E., Vanderwood, K., \& Sisson, N. (2020). Exploring a rural Latino community's perception of the COVID-19 pandemic. Ethnicity and Health. https://doi.org/10.1080/13557858.2020.1838456

Pandey, A., \& Saxena, N. K. (2020). Effectiveness of Government Policies in Controlling COVID-19 in India. International Journal of Health Services. https://doi.org/10.1177/0020731420983749

Pérez-Sastré, M. A., Valdés, J., \& Ortiz-Hernández, L. (2020). Características clínicas y gravedad de COVID19 en adultos mexicanos. Gaceta Medica de Mexico, 156(5), 373-381. https://doi.org/10.24875/GMM.M20000424

Peters, D. J. (2020). Community Susceptibility and Resiliency to COVID-19 Across the Rural-Urban Continuum in the United States. Journal of Rural Health, 36(3), 446-456. https://doi.org/10.1111/jrh.12477

Rivera-Hernandez, M., Ferdows, N. B., \& Kumar, A. (2020). The Impact of the COVID-19 Epidemic on Older Adults in Rural and Urban Areas in Mexico. The Journals of Gerontology: Series B, XX(Xx), 1-7. https://doi.org/10.1093/geronb/gbaa227

Schwalbe, N., Lehtimaki, S., \& Gutiérrez, J. P. (2020). COVID-19: rethinking risk. The Lancet Global Health, 8(8), e974-e975. https://doi.org/10.1016/S2214-109X(20)30276-X

Secretaría de Salud. (2020). Datos Abiertos Dirección General de Epidemiología. https://www.gob.mx/salud/documentos/datos-abiertos-152127

Suárez, V., Suarez Quezada, M., Oros Ruiz, S., \& Ronquillo De Jesús, E. (2020). Epidemiology of COVID19 in Mexico: From the 27th of February to the 30th of April 2020. Revista Clínica Española (English Edition), 220(8), 463-471. https://doi.org/10.1016/j.rceng.2020.05.008

Vilar-Compte, M., Pérez, V., Teruel, G., Alonso, A., \& Pérez-Escamilla, R. (2020). Costing of actions to safeguard vulnerable Mexican households with young children from the consequences of COVID-19 social distancing measures. International Journal for Equity in Health, 19(1), 4-6. https://doi.org/10.1186/s12939020-01187-3 